\title{
Near infrared VLT/MAD observations of the isolated neutron stars RX J0420.0-5022 and RX J1856.5-3754^ (Research Note)
}

\author{
R. P. Mignani ${ }^{1}$, R. Falomo ${ }^{2}$, A. Moretti ${ }^{2}$, A. Treves ${ }^{3}$, R. Turolla ${ }^{4,1}$, N. Sartore ${ }^{3}$, S. Zane ${ }^{1}$, C. Arcidiacono ${ }^{2}$, \\ M. Lombini ${ }^{5}$, J. Farinato ${ }^{2}$, A. Baruffolo ${ }^{2}$, R. Ragazzoni ${ }^{2}$, and E. Marchetti ${ }^{6}$ \\ ${ }^{1}$ Mullard Space Science Laboratory, University College London, Holmbury St. Mary, Dorking, Surrey, RH5 6NT, UK \\ e-mail: rm2@mssl.ucl.ac.uk \\ 2 INAF, Osservatorio Astronomico di Padova, Vicolo dell'Osservatorio 5, Padua 35122, Italy \\ Department of Physics and Mathematics, University of Insubria, Via Valleggio 11, Como 22100, Italy \\ 4 Department of Physics, University of Padua, via Marzolo 8, Padua 35131, Italy \\ 5 Osservatorio Astronomico di Bologna, INAF, via Ranzani 1, Bologna 40127, Italy \\ ${ }^{6}$ European Southern Observatory, Karl-Schwarzschild-Strasse 2, Garching 85748, Germany
}

Received 7 April 2008 / Accepted 16 June 2008

\section{ABSTRACT}

\begin{abstract}
Context. High-energy observations have unveiled peculiar classes of isolated neutron stars which, at variance with radio pulsars, are mostly radio silent and not powered by the star rotation. Among these objects are the magnetars, hyper-magnetized neutron stars characterized by transient X-ray/ $\gamma$-ray emission, and neutron stars with purely thermal, and in most cases stationary, X-ray emission (a.k.a., X-ray dim isolated neutron stars or XDINSs). While apparently dissimilar in their high-energy behavior and age, both magnetars and XDINSs have similar periods and unusually high magnetic fields. This suggests a tantalizing scenario where the former evolve into the latter.

Aims. Discovering so far uninvestigated similarities between the multi-wavelength properties of these two classes would be a further step forward to establish an evolutionary scenario. A most promising channels is the near infrared (NIR) one, where magnetars are characterized by a distinctive spectral flattening with respect to the extrapolation of the soft X-ray spectrum.

Methods. We observed the two XDINSs RX J0420.0-5022 and RX J1856.5-3754 with the Multi-Conjugate Adaptive Optics Demonstrator (MAD) at the Very Large Telescope (VLT), as part of the instrument guaranteed time observations program, to search for their NIR counterparts.

Results. Both RX J1856.5-3754 and RX J0420.0-5022 were not detected down to $K_{\mathrm{s}} \sim 20$ and $K_{\mathrm{s}} \sim 21.5$, respectively.

Conclusions. In order to constrain the relation between XDINSs and magnetars it would be of importance to perform deeper NIR observations. A good candidate is 1RXS J214303.7+065419 which is the XDINS with the highest inferred magnetic field.
\end{abstract}

Key words. stars: neutron

\section{Introduction}

Over the last two decades, high-energy observations have unveiled the existence of peculiar classes of isolated neutron stars (INSs) like the anomalous X-ray pulsars and the soft $\gamma$ repeaters (AXPs and SGRs), i.e., the magnetar candidates (e.g., Mereghetti 2008), and the X-ray Dim INSs (XDINSs; e.g., Haberl 2007). Despite their vastly different observational manifestations, both magnetars and XDINSs are slow rotators $(P \sim$ $1-10 \mathrm{~s})$, with surface magnetic fields reaching $\approx 10^{14}-10^{15} \mathrm{G}$ in the magnetars case, have persistent X-ray luminosities much larger than the inferred rotational energy losses $L_{\mathrm{X}} \approx 10-100 \dot{E}$, and they are usually radio-silent.

The XDINSs X-ray spectra are purely thermal and well represented by a blackbody $(k T \approx 50-100 \mathrm{eV})$ whose emission radius is consistent with a sizable fraction of the neutron star surface. In most cases, one or possibly more broad absorption features $\left(E_{\text {line }} \approx 0.2-0.7 \mathrm{keV}\right)$ have been observed (see Haberl 2007 and references therein), which hint toward magnetic fields of $\sim 10^{13}-10^{14} \mathrm{G}$, comparable to those derived from the spin

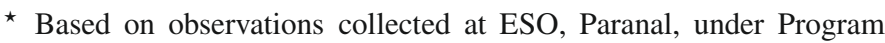
71.D-0560. down. The absence of power-law tails is consistent with the faint rotation-powered magnetospheric emission expected from the inferred spin-down luminosity $\left(\dot{E} \approx 3-5 \times 10^{30} \mathrm{erg} \mathrm{s}^{-1}\right)$. In the optical, only a few XDINSs have been identified (see Kaplan 2008 and references therein) and a candidate counterpart was recently proposed for 1RXS J214303.7+065419 (Zane et al. 2008), while that of RX J0420.0-5022 was not confirmed (Mignani et al., in preparation). Their spectra mostly follow a Rayleigh-Jeans, with fluxes exceeding by a factor $\geq 10$ the low-energy extrapolation of the X-rays best-fitting blackbody e.g., Kaplan 2008. Whether this is indeed due to emission from regions of the star surface at different temperature e.g., Pons et al. 2002 is still under debate. Like in the X-rays, no rotationpowered magnetospheric emission is detectable in the optical, unless XDINSs are a factor of $\geq 10^{3}$ more efficient emitters than rotation-powered neutron stars (Zharikov et al. 2006).

Whether there is a (evolutionary?) link between magnetars and XDINSs is still hotly debated (see McLaughlin et al. 2006; Popov et al. 2006, for a discussion). As proposed by Mignani et al. (2007a), the detection of the optical/near infrared (NIR) flattening observed in the magnetar spectra, ascribed either to magnetospheric emission powered by the magnetic field or to 
thermal emission from a fallback disk, would strengthen such a link. For the XDINSs, such flattening would be easily recognizable in the NIR, where the contribution of the optical Rayleigh-Jeans continuum is negligible. So far, NIR observations of XDINSs performed with the Very Large Telescope (VLT) did not unveil counterparts down to $\mathrm{H}=21.5-22.9$ (Lo Curto et al. 2007).

We report on new NIR observations of RX J1856.5-3754 and RX J0420.0-5022 recently performed in the $K_{\mathrm{S}}$ band with the $V L T$, the first presented for these sources. Observations are described in Sect. 2, while results are presented and discussed in Sects. 3 and 4, respectively.

\section{Observations}

\subsection{Observations description}

We performed $K_{\mathrm{s}}$-band observations of RX J1856.5-3754 and RX J0420.0-5022 on September 28 and 292007 $(M J D=54371$ and 54372), respectively, using the European Southern Observatory (ESO) Multi-Conjugate Adaptive Optics Demonstrator $(M A D)^{1}$, mounted at the Very Large Telescope (VLT) Melipal telescope, as part of the guaranteed time program. The $M A D$ instrument was developed within the European Extremely Large Telescope framework. The instrument accommodates two wavefront sensors (WFS): a star oriented (SO) multi Shack-Hartmann and a layer oriented (LO) multi pyramid (Regazzoni 2000; Regazzoni et al. 1996, 2000). Both sensors can use reference stars identified in the instrument $2^{\prime}$ field of view. The $M A D$ instrument is completed by the Camera for Multiconjugate Adaptive Optics (CAMCAO), a NIR camera equipped with a $2 K \times 2 K$ Hawaii II infrared detector movable over the $2^{\prime}$ corrected circular field-of-view. The pixel-scale is 0 .' 028 , corresponding to a $57^{\prime \prime} \times 57^{\prime \prime}$ field-of-view on the detector. Thanks to its flexibility, the instrument can be used in classical single reference adaptive optics (AO), ground layer adaptive optics (GLAO), or multi-conjugate adaptive optics (MCAO) according to the availability of suitable reference stars close to pointing direction.

During the first night, we used the LO WFS in classical AO mode, with a $V \approx 11.3$ reference star. During the second night, we used the MCAO mode with 4 fainter stars as a reference ( $V=14.9,15.3,15.4$ and 15.6). The seeing conditions during the observations were 1'.23 for RX J0420.0-5022 and 1'.37 for RX J1856.5-3754 and the airmass was 1.11 and 1.35, respectively. For RX J0420.0-5022, we obtained a total of dithered 65 exposures along a pattern that covered a region of $\sim 25^{\prime \prime} \times 25^{\prime \prime}$. The exposures were taken in blocks of 5, i.e., along each node of the dithering pattern, with $30 \mathrm{~s}$ detector integration time (DIT) and NDIT $=2$. The total integration time was $3900 \mathrm{~s}$. We performed the observation of RX J1856.5-3754 in a similar way, with a dithering pattern covering a region of $\sim 35^{\prime \prime} \times 15^{\prime \prime}$. A total of 25 exposures were obtained with $D I T=30 \mathrm{~s}$ and $N D I T=4$, for a total integration time of $3000 \mathrm{~s}$.

\subsection{Data reduction and calibration}

For each set of science images we applied standard data reduction procedures for NIR observations, which include trimming of the frames, bad pixel masking, dark and flat-field correction, and sky-background subtraction. Special care was taken for images alignment, allowing a correction for rotation to account for

${ }^{1}$ http://www.eso.org/projects/aot/mad/ a non-optimal efficiency of the de-rotator. For the flat-field correction, we used the median of several images obtained on the sky at the beginning of each night. The bad pixel mask was created by analyzing the ratio of two flat-field images with significantly different exposure levels. For each science observation we then constructed a reference sky image. Then we constructed the reference sky image from the median of all science images of a given observation. In the case of our targets, the fields are not affected by crowding of stellar sources, which allows for the creation of a good quality sky image. This was then normalized to the median counts of each science image and subtracted. Finally, we combined all sky-subtracted science images after properly aligning them using as a reference the positions of the brightest (not saturated) sources detected in all images.

From the final co-added images we measured the associated image quality from the average full width at half maximum (FWHM) of the intensity profile of a number of reference stars. Although the nominal seeing conditions in the night were not optimal (see above), thanks to the MAD AO correction, the FWHM of stellar images measured on the detector actually turned out to be 0.28 and 0. '40 for the RX J0420.0-5022 and for the RX J1856.5-3754 field, respectively. We performed the photometric calibration by observing NIR standard stars during the same nights. We cross-checked our calibration by direct comparison with objects of the $2 M A S S$ point-source catalog (Skrutskie et al. 2006).

\section{Data analysis and results}

\subsection{Astrometry}

The astrometry of the MAD images of RX J1856.5-3754 was computed using as a reference the coordinates and position of 8 2MASS stars (Skrutskie et al. 2006), all non-saturated and evenly distributed in the field. Their pixel coordinates were measured by fitting their intensity profiles with a Gaussian function using the Graphical Astronomy and Image Analysis (GAIA) interface ${ }^{2}$. The coordinate transformation between the detector and the celestial reference frame was then computed using the Starlink package ASTROM ${ }^{3}$. The rms of the astrometric solution was $\approx 0$ '! 17 . After accounting for a 0 '. 2 conservative astrometric accuracy of $2 M A S S$, and the $\sim 15$ mas accuracy (Skrutskie et al. 2006) on the tie to the International Celestial Reference Frame (ICRF), the overall error to be attached to our astrometry was finally 0.26 . As a reference for the RX J1856.5-3754 coordinates at the epoch of the MAD observation (2007.74) we used those of its optical counterpart reported in Kaplan et al. (2002), epoch 1999.26, corrected for the measured proper motion. The uncertainty to be attached to the 2007.74 coordinates includes the error on the proper motion extrapolation between the two epochs, $\sim 9$ mas both in right ascension and declination, and the error on the reference coordinates which is 0.2 relative to the ICRF. We then computed the overall uncertainty on the position determination of RX J1856.5-3754 on the MAD images by adding in quadrature the error on our astrometric calibration and

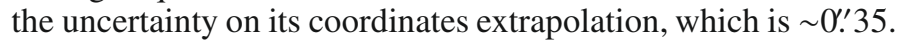

Since no $2 M A S S$ star is identified in the much less crowded RX J0420.0-5022 field, we performed a two-step astrometric calibration using as a reference a grid of stars selected from the VLT H-band ISAAC image of the field (Lo Curto et al. 2007). By applying the same procedure as before, the astrometric calibration of the ISAAC image was performed using $82 M A S S$ stars as a

\footnotetext{
2 star-www.dur.ac.uk/ pdraper/gaia/gaia.html

3 http://star-www.rl.ac.uk/Software/software.htm
} 


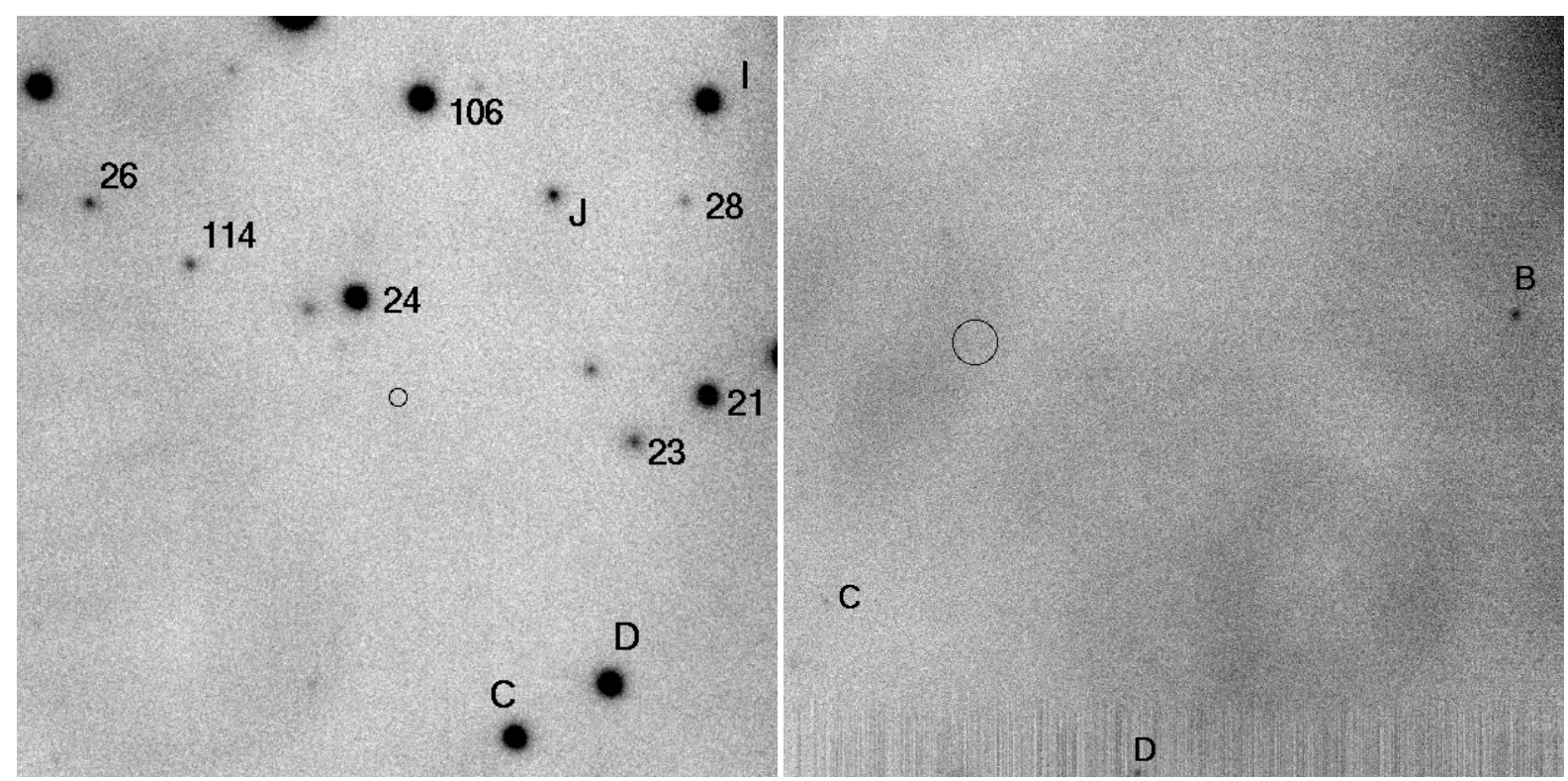

Fig. 1. We present $30^{\prime \prime} \times 30^{\prime \prime} M A D K_{\mathrm{s}}$-band images of the RX J1856.5-3754 (left) and RX J0420.0-5022 (right) fields. North to the top, East to the left. The circles mark the expected target positions, with the radii ( 0.35 and $1^{\prime \prime}$, respectively) corresponding to their positional uncertainty (see Sect. 3.1). The labeling in the RX J1856.5-3754 field is the same used in van Kerkwijk \& Kulkarni (2001). The few faint objects detected in the RX J0420.0-5022 field are also labeled. The intensity scale in the image has been adjusted for a better visualization. The edge of the bright field galaxy ESO 202-8 is visible in the top right of the image.

reference, yielding an rms of $\sim 0$ ' 22 on the astrometric solution. We then computed the coordinates of 7 field stars in common between the ISAAC and MAD fields, which made our secondary astrometric grid. The astrometric solution yields an rms of $\sim 0$ ' $^{\prime} 09$. By adding in quadrature all the errors of our astrometric chain, we then end up with an accuracy of 0.32 on our astrometry. As a reference for the RX J0420.0-5022 coordinates, we used the Chandra coordinates of Haberl et al. (2004), which have a nominal error of 0.'6 (90\% confidence level). We accounted for the recently measured proper motion upper limit (138 mas $\mathrm{yr}^{-1}$; Motch et al. 2007) when we extrapolated the Chandra coordinates of RX J0420.0-5022 (epoch 2002.86) to the epoch of the $M A D$ observation (2007.74). This yields a position uncertainty due to the proper motion of $\sim 0.7$. By adding in quadrature the error on our astrometric calibration and the error on the original Chandra coordinates we ended up with an uncertainty of $\sim 1^{\prime \prime}$ on the RX J0420.0-5022 position at the epochs of the MAD observations.

The two panels in Fig. 1 show a zoom of the MAD image around the expected position of RX J1856.5-3754 and RX J0420.0-5022. Despite of the sharper PSF of MAD, our images do not unveil any new object with respect to the ones identified in the long-exposure ISAAC $\mathrm{H}$-band images of Lo Curto et al. (2007). In both cases, no candidate counterpart is detected at the expected position, with the closest objects located several arcseconds away.

\subsection{Photometry}

To determine whether our observations can be used to better constrain the NIR emission properties of RX J1856.5-3754 and RX J0420.0-5022, we estimated their $K_{\mathrm{s}}$-band brightness upper limits from the limiting magnitudes of the $M A D$ images. To this aim, we have used as a reference the flux of a number of artificial stars simulated with the template PSF measured on the images. Using different test values for the PSF flux normalization, artificial stars were simulated and added to the corresponding images at different coordinates, randomly selected within a few arcsec from the target position. We then ran in loop the Daophot detection tool to determine the PSF flux normalization corresponding to a $3 \sigma$ detection limit. In all cases, aperture correction was applied. We thus determined that $3 \sigma$ detections would correspond to magnitudes of $K_{\mathrm{S}} \sim 21.5$ and $K_{\mathrm{S}} \sim 20$ for RX J0420.0-5022 and RX J1856.5-3754, respectively. As an independent check, we have compared these values with the $3 \sigma$ detection limits extrapolated from the fluxes of the faintest objects detected in our images. As a safe measure, we used the ISAAC $H$-band images of Lo Curto et al. (2007) to confirm that they were indeed genuine detections and not background fluctuations. Following this empirical approach, we derived detection limits consistent with the previous ones. The different limiting magnitudes for the two images, despite comparable integration times, has to be ascribed mainly to the different image quality between the two frames, being much better for RX J0420.0-5022 than for RX J1856.5-3754 (0.'28 with respect to 0.'40, see Sect. 2.2). This is indeed a significant factor when the signal-to-noise ratio is background limited. Moreover, the RX J1856.5-3754 image is affected by a higher background level which is $~ 25 \%$ higher around the target position.

\section{Discussion}

Our measured $K_{\mathrm{s}}$-band spectral flux upper limits are well above the extrapolation of the optical spectrum of RX J1856.5-3754 (van Kerkwijk \& Kulkarni 2001), which would predict $K_{\mathrm{S}} \sim$ 26.5, and the Rayleigh-Jeans extrapolation in the optical/NIR domain of the XMM-Newton spectrum of RX J0420.0-5022 (Haberl et al. 2004). We note that, for RX J1856.5-3754, the $K_{\mathrm{s}}$-band spectral flux upper limit is shallower than the $H$-band one of Lo Curto et al. (2007), while for RX J0420.0-5022 it is slightly deeper. Thus, we are not yet able to constrain the NIR spectrum of RX J1856.5-3754 and RX J0420.0-5022.

A blackbody spectrum produced by the neutron star surface is obviously a possibility. It is clear that for the 

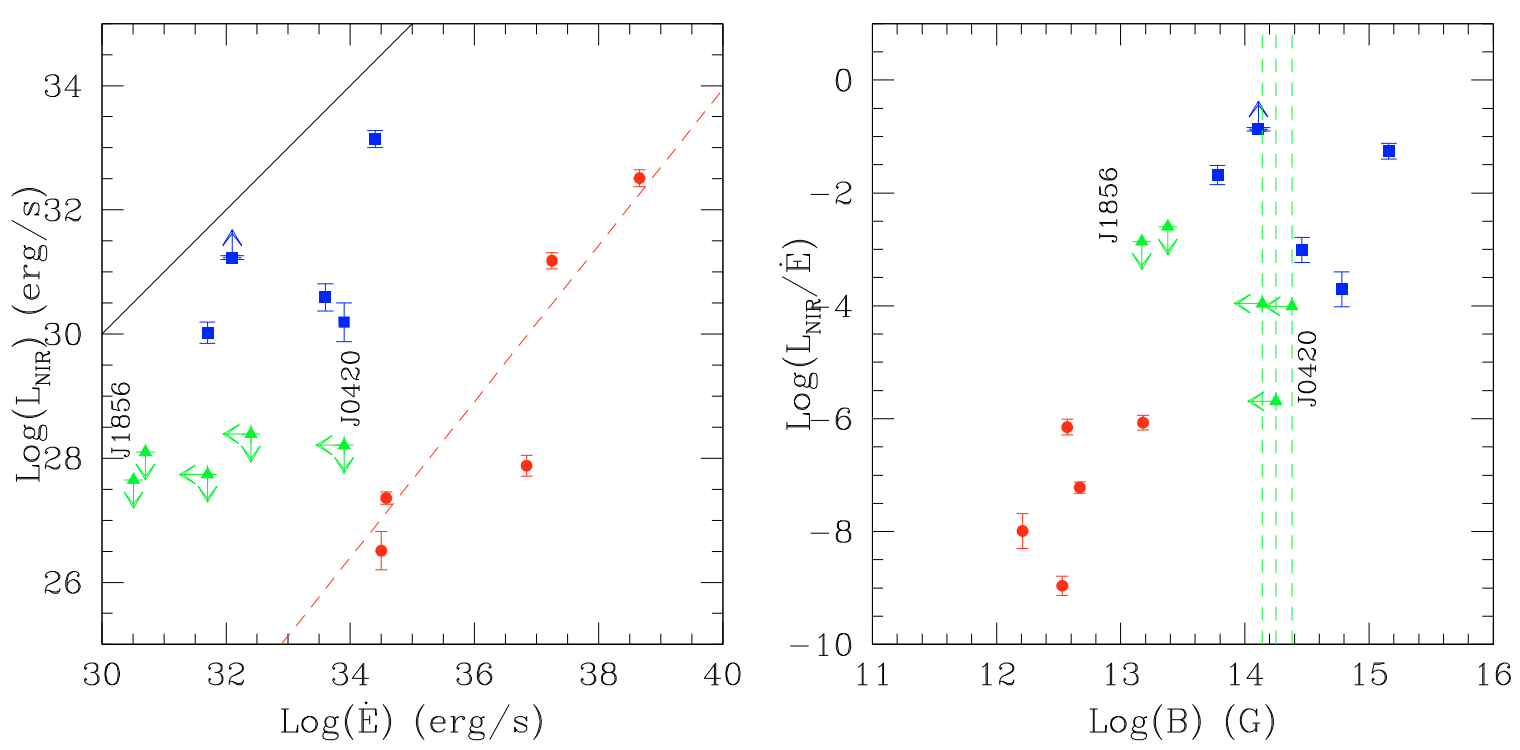

Fig. 2. (left) INS NIR luminosities vs. rotational energy loss $\dot{E}$. Filled circles, squares and triangles correspond to rotation-powered neutron stars, magnetars, and XDINSs, respectively. Plots are updated from Mignani et al. (2007b) and Lo Curto et al. (2007), to which we refer for the complete source list and for the timing parameters. Luminosities are computed in the $K_{\mathrm{s}}$ band for magnetars and for rotation-powered neutron stars. For the XDINSs, we have plotted the luminosity upper limits computed from the $H$-band observations of Lo Curto et al. (2007). For RX J0420.0-5022 only, we have plotted the slightly deeper luminosity upper limit obtained from our $K_{\mathrm{s}}$-band observations. The red dashed line is the linear fit to rotation-powered neutron stars, while the solid line marks the limit case $\log \left(L_{\text {NIR }}\right)=\log (\dot{E})$. $(r i g h t) \operatorname{INS}$ NIR efficiencies, $\eta_{\text {NIR }} \equiv L_{\text {NIR }} / \dot{E}$, vs. dipole magnetic field $B$ inferred from the spin down. Note that for those XDINSs for which only an upper limit on $\dot{E}$ is available, including RX J0420.0-5022, the NIR emission efficiency is unconstrained, as shown by the vertical dashed lines.

RX J1856.5-3754 and RX J0420.0-5022 distances of $\sim 160 \mathrm{pc}$ (van Kerkwijk \& Kaplan 2007) and 350 pc (Posselt et al. 2007), respectively, and for any reasonable combination of temperature and emitting area, the predicted flux would fall well below our upper limits. A blackbody spectrum produced by a fallback disk (Perna et al. 2000) could yield to a flux compatible with the present NIR flux upper limits of RX J1856.5-3754 and RX J0420.0-5022, depending on the actual disk size and accretion rate (see also Lo Curto et al. 2007). A power-law spectrum could be produced by non-thermal emission from the neutron star magnetosphere. In Fig. 2 (left), we plotted the NIR luminosity $\left(L_{\mathrm{NIR}}\right)$ vs the rotational energy loss $(\dot{E})$ for different classes of INSs: rotation-powered neutron stars, magnetars, and XDINSs. Our upper limits do not rule out a NIR emission efficiency, $\eta_{\mathrm{NIR}} \equiv L_{\mathrm{NIR}} / \dot{E}$, comparable to that of rotation-powered neutron stars, or higher. This would be possible, for instance, if the fraction of energy emitted in the relativistic wind is much less than in rotation-powered neutron stars or if the NIR emission is powered not by the neutron star rotation but, e.g., by its magnetic field. Although XDINSs are less luminous than magnetars in absolute terms (Fig. 2, left), they might have a comparable NIR emission efficiency. This is shown in Fig. 2 (right) where the NIR emission efficiency is plotted vs. the dipole magnetic field $B$ inferred from the spin down. We warn here that NIR observations of the radio pulsar PSR J1119-6127 (Mignani et al. 2007b) suggest a NIR emission efficiency comparable to that of other rotation-powered neutron stars, despite of its relatively high magnetic field $\left(B \sim 4.1 \times 10^{13} \mathrm{G}\right)$. If the inferred magnetic field value of PSR J1119-6127 is correct, this might argue against a connection between the NIR emission efficiency and the magnetic field.

Much deeper observations are required to better constrain the XDINS optical/NIR emission properties and to investigate their possible connection with the magnetars. In this respect, particularly important would be the study of 1RXS J214303.7+065419, possibly the XDINS with the highest magnetic field, as hinted by the detection of the X-ray absorption feature (Zane et al. 2005). Its anomalously high optical emission is indeed incompatible with both thermal emission from the neutron star surface and rotation-powered non-thermal emission (Zane et al. 2008).

Acknowledgements. RPM acknowledges STFC for support through a rolling grant and the ESO/Chile Visitors Program for hospitality at the Santiago Office where most of this work was finalized. RPM also thanks P. Amico and G. Lo Curto (ESO) for useful discussions. SZ acknowledges STFC for support through an Advanced Fellowship.

\section{References}

Camilo, F., Ransom, S. M., Halpern, J. P., et al. 2006, Nature, 442, 892 Camilo, F., Ransom, S. M., Halpern, J. P., \& Reynolds, J. 2007, ApJ, 666, L93 Fitzpatrick, E. L. 1999, PASP, 111, 63

Haberl, F. 2007, Ap\&SS, 308, 181

Haberl, F., Motch, C., Zavlin, V. E., et al. 2004, A\&A, 424, 635

Kaplan, D. L. 2008, Proc. of "40 Years of Pulsars: Millisecond Pulsars, Magnetars, and More", AIP, 983, 331

Lo Curto, G., Mignani, R. P., Perna, R., et al. 2007, A\&A 473, 539

McLaughlin, M. A., Lyne, A. G., Lorimer, D. R., et al. 2006, Nature, 439, 817

Mereghetti, S. 2008, A\&ARv, in press, [arXiv:0804.0250]

Mignani, R., Bagnulo, S., de Luca, A., et al. 2007a, AP\&SS, 308, 203

Mignani, R. P., Perna, R., Rea, N., et al. 2007b, A\&A, 471, 265

Motch, C.,Pires, A. M., Haberl, F., \& Schwope, A. 2007, Ap\&SS, 308, 217

Perna, R., Hernquist, L., \& Narayan, R. 2000, ApJ, 541, 344

Pons, J. A., Walter, F. M., Lattimer, J. M., et al. 2002, ApJ, 564, 981

Popov, S. B., Turolla, R., \& Possenti, A. 2006, MNRAS, 369, L23

Posselt, B., Popov, S. B., Haberl, F., et al. 2007, Ap\&SS, 308, 171

Ragazzoni, R. 1996, J. Mod. Opt., 43, 289

Ragazzoni, R. 2000, Proc. of "Backaskog workshop on extremely large telescopes", 57, 175

Ragazzoni, R., Farinato, J., \& Marchetti, E. 2000, Spie, 4007, 1076

van Kerkwijk, M. H., \& Kulkarni, S. R. 2001, A\&A, 378, 986

van Kerkwijk, M. H., \& Kaplan, D. L. 2007, Ap\&SS, 308, 191

Zane, S., Cropper, M., Turolla, R., et al. 2005, ApJ, 627, 397

Zane, S., Mignani, R. P., Turolla, R., et al. 2008, ApJ, accepted [arXiv:0804.4394]

Zharikov, S., Shibanov, Yu., \& Komarova, V., 2006, AdSpR, 37, 1979 